Discussion Paper No. 668

\title{
DOMAINS OF SOCIAL CHOICE FUNCTIONS ON WHICH COALITION STRATEGY-PROOFNESS AND MASKIN MONOTONICITY ARE EQUIVALENT
}

Koji Takamiya

July 2006

The Institute of Social and Economic Research Osaka University

6-1 Mihogaoka, Ibaraki, Osaka 567-0047, Japan 


\title{
Domains of social choice functions on which coalition strategy-proofness and Maskin monotonicity are equivalent*
}

\author{
Koji TAKAMIYA ${ }^{\dagger}$ \\ Institute of Social and Economic Research, \\ Osaka University
}

29 June 2006

\begin{abstract}
It is known that on some social choice and economic domains, a social choice function is coalition strategy-proof if and only if it is Maskin monotonic (e.g. Muller and Satterthwaite, 1977). This paper studies the foundation of those results. I provide a set of conditions which is sufficient for the equivalence between coalition strategy-proofness and Maskin monotonicity. This generalizes some known results.

JEL Classification- C71, C72, D71.

Keywords - social choice function, coalition strategy-proofness, Maskin monotonicity.
\end{abstract}

\section{Introduction}

This paper examines logical relations between coalition strategy-proofness and Maskin monotonicity of social choice functions. Coalition strategy-proofness is a strong requirement of incentive compatibility. A social choice function is said to be coalition strategy-proof if no group of individuals can benefit from jointly misrepresenting their preferences, in other words, cannot manipulate the final outcome. A social choice function is said to be Maskin monotonic if the outcome to be chosen by the function does not vary whenever each individual switches his preference keeping or improving the relative ranking of that outcome. This property is very important in implementation theory. For example, it is well-known as a necessary condition for Nash implementation (see Maskin, 1985, 1999).

It has been observed that these two properties are strongly related to each other. The classical result by Muller and Satterthwaite (1977) asserts that on the

\footnotetext{
${ }^{*}$ Part of the research has been done while I visited to the Indian Statistical Institute, Delhi Centre. I am very grateful to Arunava Sen for his hospitality and helpful comments. And I thank the people of the institute for their hospitality. All errors are my own responsibility.

${ }^{\dagger}$ Institute of Social and Economic Research, Osaka University. 6-1 Mihogaoka Ibaraki Osaka 567-0047 JAPAN. tel: +81-6-6879-8556. E-mail: takamiya@iser.osaka-u.ac.jp.
} 
unrestricted strict preference domain, a social choice function is strategy-proof if and only if it is Maskin monotonic. ${ }^{1}$ Since on this domain, strategy-proofness is equivalent to coalition strategy-proofness, the theorem states the equivalence between coalition strategy-proofness and Maskin monotonicity.

In more recent studies (e.g. Svensson, 1999; Takamiya 2001, 2003), it has been pointed out that coalition strategy-proofness and Maskin monotonicity are equivalent for allocation rules in a certain broad class of economies with indivisible goods, which includes some notable problems such as "marriage problems" (Gale and Shapley, 1962) and "housing markets" (Shapley and Scarf, 1974). Also in some other environments, for example the classical exchange economies, it is known that coalition strategy-proofness implies Maskin monotonicity (e.g. Barberà and Jackson, 1995).

The purpose of this paper is to study the foundation of these close relationships between coalition strategy-proofness and Maskin monotonicity. I examine what conditions the domain of the social choice function should satisfy in order to have the property that coalition strategy-proofness implies Maskin monotonicity, and its converse.

The main results of this paper provide two sufficient conditions. The first condition, which is referred to as Condition A, is a sufficient condition for that coalition strategy-proofness implies Maskin monotonicity. This condition requires the domain of the social choice function to satisfy two properties. The first property says that for any individual, and any preference admissible to him, if any two alternatives are indifferent under this preference, then these alternatives are indifferent under all the preferences admissible to him. In other words, this requires that every individual has a partition of the set of alternatives, and his admissible preferences contains only (but not necessarily all of) such preferences that any two alternatives are indifferent if, and only if, these alternatives are in the same cell of the partition. The second property that Condition A requires is that if for any preference profile in the domain, there is no pair of alternatives such that all individuals are indifferent between them. That is to say, if the alternative to be chosen shifts from one to another, then there is always someone who cares about this shift. This is equivalent to the requirement that the "join" (the coarsest common refinement) of the partitions of all the individuals that arise in the first half of this condition equals to the finest partition (i.e. the one in which each cell contains exactly one element).

The second condition, referred to as Condition B, is a sufficient condition for that Maskin monotonicity implies coalition strategy-proofness. This condition is defined as follows: Let any coalition be given. And pick up any preference profile for this coalition, which I call the first profile. Then let us fix any two alternatives, say $x$ and $y$, such that $y$ (weakly) Pareto dominates $x$ within this coalition under the first profile. Further, pick up another arbitrary preference profile for this coalition, the second profile. Then this domain satisfies Condition B if the domain contains at least one preference profile for this coalition such that $x$ keeps or improves its

\footnotetext{
1 "Strategy-proofness" requires that the social choice function cannot be manipulated by any single individual. That is, unlike coalition strategy-proofness, manipulations by groups are not necessarily ruled out.
} 
relative ranking from the first profile to this profile, and so does $y$ from the second profile to this profile. Speaking very roughly, the third profile is a mixture of the first and the second profiles in the sense of the desirability of $x$ and $y$. And Condition $\mathrm{B}$ requires such a mixture always exists.

Given these two sufficient conditions, we present a class of domains which satisfies both of these conditions. This class is the collection of those domains in which (i) every individual has a partition of the set of alternatives, and his admissible preferences are exactly such preferences that any two alternatives are indifferent if, and only if, these alternatives are in the same cell of the partition, and (ii) the join of all these partitions equals to the finest partition. Paraphrasing, such a domain is the maximal domain among those satisfying Condition A, given a list of partitions. I call these domains essentially strict domains.

I point out that essentially strict domains are assumed in some previous results. This observation unifies the the Muller-Satterthwaite theorem and the similar equivalence theorem by Takamiya (2003) in the context of the "generalized indivisible good allocation problems" (Sönmez, 1999), which cover various problems including well-known "housing markets" (Shapley and Scarf, 1974) and "marriage problems" (Gale and Shapley, 1962).

\section{Preliminaries}

Let $N$ denote the set of individuals. Assume that $N$ is a nonempty finite set. Call any nonempty subset of $N$ a coalition. Let $X$ be the set of alternatives (social outcomes). $X$ is nonempty and may be finite or infinite.

Let $Q$ be a nonempty set. Then denote by $W(Q)$ the set of weak orderings (i.e. complete and transitive binary relations) on $Q$. And denote by $L(Q)$ the set of linear orderings (i.e. complete, tansitive and anti-symmetric binary relations) on $Q$.

For $i \in N$, call $R^{i} \in W(Q)$ a preference relation on $Q$ of individual $i$. And a list $\left(R^{i}\right)_{i \in N}$ is called a preference profile. For $x, y \in Q, x R^{i} y$ reads that to individual $i, x$ is at least as good as $y$. As usual, $P^{i}$ denotes the asymmetric part, and $I^{i}$ denotes the symmetric part of $R^{i}$. Let $R^{i} \in W(Q)$ and $Q^{\prime} \subset Q$. Then $\max R^{i}(Q)$ denotes the set of $R^{i}$-maximal elements in $Q^{\prime},\left\{x \in Q^{\prime} \mid \forall y \in Q^{\prime}, x R^{i} y\right\}$.

For $i \in N, D^{i}$ denotes the set of admissible preferences of individual $i$. Assume that $D^{i} \subset W(X)$ for any $i \in N$. For $S \subset N, D^{S}$ denotes the Cartesian product $\prod_{i \in S} D^{i}$. A social choice function (SCF) is a single-valued function $f: D^{N} \rightarrow X$. $D^{N}$ is called the domain (of $f$ ).

Let $f$ be a SCF. Let $S$ be a coalition. Then we say that $S$ manipulates $f$ at a preference profile $R \in D^{N}$ if there exists some $R^{\prime S} \in D^{S}$ such that

$$
\left[\forall i \in S, f\left(R^{-S}, R^{\prime S}\right) R^{i} f(R)\right] \&\left[\exists j \in S: f\left(R^{-S}, R^{\prime S}\right) P^{j} f(R)\right] .
$$

Call $f$ coalition strategy-proof if no coalition manipulates $f$ at any $R \in D^{N}$.

For $R^{i} \in W(X)$, and $x \in X$, denote by $L\left(x, R^{i}\right)$ the set $\left\{y \in X \mid x R^{i} y\right\}$. That is, $L\left(x, R^{i}\right)$ is the lower-contour set of $x$ relative to $R^{i}$. Call $f$ Maskin monotonic 
if for any $R, R^{\prime} \in D^{N}$,

$$
\left[f(R)=x \&\left(\forall i \in N, L\left(x, R^{i}\right) \subset L\left(x, R^{\prime i}\right)\right)\right] \Rightarrow f\left(R^{\prime}\right)=x .
$$

\section{Main results}

This section presents the main results. These results provide sufficient conditions that the domain of the social choice function should satisfy to have the property that coalition strategy-proofness implies Maskin monotonicity, and its converse. I introduce these two sufficient conditions. Let a SCF $f: D^{N} \rightarrow X$ be given.

Let $\mathcal{P}^{i}$ be a partition of $X$. For $x \in X$, let us denote the cell of $\mathcal{P}^{i}$ that contains $x$ by $\mathcal{P}^{i}(x)$. Let $\left(\mathcal{P}^{i}\right)_{i \in N}$ be a profile of partitions of $X$. And let us denote by "V" the operation of taking the "join" (the coarsest common refinement) of the partitions.

Conditon A. There exists some profile of partitions $\left(\mathcal{P}^{i}\right)_{i \in N}$ such that for any $R \in D^{N}$, any $i \in N$ and any $x, y \in X$,

$$
x \in \mathcal{P}^{i}(y) \Leftrightarrow x I^{i} y
$$

and

$$
\bigvee_{i \in N} \mathcal{P}^{i}=\{\{x\} \mid x \in X\}
$$

In words, Condition A consists of two parts, which correspondence to the formulas (3) and (4), respectively:

(i) For any individual, and for any preference admissible to him, any two alternatives are indifferent under this preferences if, and only if, these alternatives are indifferent under all the preferences admissible to him; and

(ii) If for any preference profile, for any pair of alternatives there is at least one individual who is not indifferent between these alternatives.

Condition B. For any $S \subset N$ with $S \neq \emptyset$, any $\tilde{R}^{S}, \hat{R}^{S} \in D^{S}$, and any $x, y \in X$ such that $\left(\forall i \in S, y \tilde{R}^{i} x\right)$ and $\left(\exists i \in S: y \tilde{P}^{i} x\right)$, there exists $R^{\star S} \in D^{S}$ such that

$$
\forall i \in S, L\left(x, \tilde{R}^{i}\right) \subset L\left(x, R^{\star i}\right) \& L\left(y, \hat{R}^{i}\right) \subset L\left(y, R^{\star i}\right) .
$$

In words, Condition B condition is defined as follows: Let any coalition be given. And pick up any preference profile for this coalition, which I call the first profile. Then let us fix any two alternatives, say $x$ and $y$, such that $y$ (weakly) Pareto dominates $x$ within this coalition under the first profile. Further, pick up another arbitrary preference profile for this coalition, the second profile. Then the domain satisfies Condition B if the domain contains at least one preference profile for this coalition such that $x$ keeps or improves its relative ranking from the first profile to this profile, and so does $y$ from the second profile to this profile. Roughly speaking, the third profile is a mixture of the first and the second profiles in the sense of the desirability of $x$ and $y$. And the condition requires such a mixture always exists. 
Theorem 1 Let $D^{N}$ satisfy Condition A. Then if $f$ is coalition strategy-proof, then $f$ is Maskin monotonic.

Proof Suppose that $D^{N}$ satisfies Condition A and that $f$ is not Maskin monotonic. Then I will show that $f$ is not coalition strategy-proof. Since $f$ is not Maskin monotonic, we have for some $i \in N$, some $R \in D^{N}$, and some $\tilde{R}^{i} \in D^{i}$,

$$
L\left(x, R^{i}\right) \subset L\left(x, \tilde{R}^{i}\right) \& f\left(R^{-i}, \tilde{R}^{i}\right) \neq x,
$$

where $x$ denotes the alternative $f(R)$. Let us denote $f\left(R^{-i}, \tilde{R}^{i}\right)$ by $y$. Since $D^{N}$ satisfies Condition A, there must be at least one individual $j$ such that $x P^{j} y$ or $y P^{j} x$. Thus the set $T=\left\{j \in N \mid \neg x I^{j} y\right\}$ is nonempty. Then there are two cases.

(i) Assume that $i \in T$. Then either $x P^{i} y$ or $y P^{i} x$. Suppose that $x P^{i} y$ holds true. Then $L\left(x, R^{i}\right) \subset L\left(x, \tilde{R}^{i}\right)$ and Condition A together imply $x \tilde{P}^{i} y$. That is, $f\left(R^{-i}, R^{i}\right) \tilde{P}^{i} f\left(R^{-i}, \tilde{R}^{i}\right)$, which says $i$ manipulate at $\left(R^{-i}, \tilde{R}^{i}\right)$ by reporting $R^{i}$. Thus $f$ is not coalition strategy-proof.

In turn, suppose that $y P^{i} x$ holds true. Then similarly, $i$ manipulate at $R$ by reporting $\tilde{R}^{i}$, which violates coalition strategy-proofness again.

(ii) Assume that $i \notin T$. Then $x I^{i} y$. Let $j \in T$, which means either $x P^{j} y$ or $y P^{j} x$. Suppose that $x P^{j} y$. Then similarly to the case (i), $\{i, j\}$ manipulates at $\left(R^{-\{i, j\}}, \tilde{R}^{i}, R^{j}\right)$ by reporting $\left(R^{i}, R^{j}\right)$. In turn suppose that $y P^{j} x$. Then $\{i, j\}$ manipulates at $\left(R^{-\{i, j\}}, R^{i}, R^{j}\right)$ by reporting $\left(\tilde{R}^{i}, R^{j}\right)$. In either way, $f$ is not coalition strategy-proof.

Theorem 2 Let $D^{N}$ satisfy Condition B. Then if $f$ is Maskin monotonic, then $f$ is coalition strategy-proof.

Proof Suppose that $D^{N}$ satisfies Condition B and that $f$ is Maskin monotonic but not coalition strategy-proof. Then there is some coalition $S$ which manipulates at some $\tilde{R} \in D^{N}$ by reporting $\hat{R}^{S} \in D^{S}$. Let us denote the alternative $f(\tilde{R})$ by $x$, and $f\left(\tilde{R}^{-S}, \hat{R}^{S}\right)$ by $y$. Then clearly, $\forall i \in S, y \tilde{R}^{i} x$ and $\exists i \in S: y \tilde{P}^{i} x$. Thus Condition B implies that there is $R^{\star S} \in D^{S}$ such that for all $i \in S$,

$$
\begin{gathered}
L\left(x, \tilde{R}^{i}\right) \subset L\left(x, R^{\star i}\right), \\
L\left(y, \hat{R}^{i}\right) \subset L\left(y, R^{\star i}\right) .
\end{gathered}
$$

Since $f$ is Maskin monotonic, (6) implies $f\left(\tilde{R}^{-S}, R^{\star S}\right)=x$. On the other hand, (7) implies $f\left(\tilde{R}^{-S}, R^{\star S}\right)=y$. These imply $x=y$, a contradiction.

\section{$3 \quad$ Further results}

This section provides a class of domains which satisfies both Conditions A and B. Then applying the results presented in Section 2, some previous results will be derived. 
Essentially strict domain. Let $\left(\mathcal{P}^{i}\right)_{i \in N}$ be a profile of partitions of $X$. Then $D^{N}$ is said to be the essentially strict domain with respect to $\left(\mathcal{P}^{i}\right)_{i \in N}$ if $D^{N}$ is the collection of all the preference profiles $R$ that satisfy for any $x, y \in X$,

$$
x \in \mathcal{P}^{i}(y) \Leftrightarrow x I^{i} y
$$

and $\left(\mathcal{P}^{i}\right)_{i \in N}$ satisfies

$$
\bigvee_{i \in N} \mathcal{P}^{i}=\{\{x\} \mid x \in X\}
$$

To paraphrase, the essentially strict domain with respect to $\left(\mathcal{P}^{i}\right)_{i \in N}$ is the (inclusion) maximal domain among those which satisfy Condition A given $\left(\mathcal{P}^{i}\right)_{i \in N}$.

It is less obvious that such a domain satisfies Condition B.

Lemma 1 If $D^{N}$ is the essentially strict domain with respect to $\left(\mathcal{P}^{i}\right)_{i \in N}$, then $D^{N}$ satisfies Condition $B$.

Proof Let $S \subset N$ with $S \neq \emptyset$, and $x, y \in X$. Let $\tilde{R}^{S} \in D^{S}$ such that $(\forall i \in$ $\left.S, y \tilde{R}^{i} x\right) \&\left(\exists i \in S, y \tilde{P}^{i} x\right)$, and $\hat{R}^{S} \in D^{S}$. To show that $D^{N}$ satisfies Condition $\mathrm{B}$, we will give $R^{\star S} \in D^{S}$ such that

$$
\forall i \in S, L\left(x, \tilde{R}^{i}\right) \subset L\left(x, R^{\star i}\right) \& L\left(y, \hat{R}^{i}\right) \subset L\left(y, R^{\star i}\right) .
$$

Let $S^{+}=\left\{i \in S \mid y \tilde{P}^{i} x\right\}$, and $S^{0}=\left\{i \in S \mid y \tilde{I}^{i} x\right\}$. Let $R^{\star S}$ be such that for each $i \in S^{+}$,

$$
\begin{array}{r}
\max R^{\star i}(X)=\mathcal{P}^{i}(y), \text { and } \\
\max R^{\star i}\left(X \backslash \max R^{\star i}(X)\right)=\mathcal{P}^{i}(x),
\end{array}
$$

and for each $i \in S^{0}$,

$$
\max R^{\star i}(X)=\mathcal{P}^{i}(y) .
$$

Note that for $i \in S^{0}, \mathcal{P}^{i}(x)=\mathcal{P}^{i}(y)$. Then evidently, $L\left(y, R^{\star i}\right)=X$ for any $i \in S$; $L\left(x, R^{\star i}\right)=X$ for any $i \in S^{0}$; and $L\left(x, R^{\star i}\right)=X \backslash \mathcal{P}^{i}(y)$ for any $i \in S^{+}$. Note that $L\left(x, \tilde{R}^{i}\right) \cap \mathcal{P}^{i}(y)=\emptyset$. Thus $R^{\star S}$ satisfies (11).

Now we obtain the following result applying Theorems 1 and 2 .

Theorem 3 Let $D^{N}$ be an essentially strict domain. Then $f$ is coalition strategyproof if, and only if, $f$ is Maskin monotonic.

In the following, we will derive two known results as special cases of Theorem 3. First, let the partition profile $\left(\mathcal{P}^{i}\right)_{i \in N}$ be such that for each $i, \mathcal{P}^{i}$ is $\{\{x\} \mid x \in X\}$. Then the essentially strict domain with respect to $\left(\mathcal{P}^{i}\right)_{i \in N}$ coincides with $L(X)^{N}$. This yields the well-known Muller-Satterthwaite theorem.

Corollary 1 (Muller and Satterthwaite, 1977) Let $D^{N}=L(X)^{N}$. Then $f$ is coalition strategy-proof if, and only if, it is Maskin monotonic. 
The second application is for "generalized indivisible good allocation problems," as defined in Sönemz (1999). This class of allocation problems contains well-known "marriage problems" (Gale and Shapley, 1962) and "housing markets" (Shapley and Scarf, 1974) as special cases. A generalized indivisible good allocation problem (an allocation problem, henceforth) is a list $(N, \Omega, \mathcal{A}, R) .{ }^{2}$ Here $N$ is the set of individuals, as we have defined in Section $1 . \Omega$ is the set of goods, which is assumed to be a nonempty finite set. An "allocation" is a set-valued function $x: N \rightarrow \rightarrow \Omega$ such that $\{x(i) \mid i \in N\}$ is a partition of $\Omega$. $\mathcal{A}$ is the set of feasible allocations. And $R$ is a preference profile belonging to the domain $D^{N}$ defined as follows:

$$
D^{N}:=\left\{R \mid \forall i \in N, R^{i} \in W(\mathcal{A}) \&\left(\forall x, y \in \mathcal{A}, x I^{i} y \Leftrightarrow x(i)=y(i)\right)\right\} .
$$

That is, every individual has preferences that exhibit no consumption externalities, and are strict over their own assignments.

Obviously, in this case, $D^{N}$ is an essentially strict domain with respect to $\left(\mathcal{P}^{i}\right)_{i \in N}$, where for each $i \in N, \mathcal{P}^{i}$ is the partition such that for any $x, y \in \mathcal{A}$, $x \in \mathcal{P}(y) \Leftrightarrow x(i)=y(i)$.

In this setting, we consider the set of allocation problems $\{(N, \Omega, \mathcal{A}, R) \mid R \in$ $\left.D^{N}\right\}$, and SCFs $f: D^{N} \rightarrow \mathcal{A}$. Then we have the following known result as a corollary to Theorem 3 .

Corollary 2 (Takamiya, 2003) Let $f$ be a SCF in a setting of allocation problems. Then $f$ is coalition strategy-proof if, and only if, it is Maskin monotonic.

\section{References}

[1] Barberà S and Jackson MO (1995) Strategy-proof exchange. Econometrica 63: pp5187

[2] Gale D and Shapley L (1962) College admissions and the stability of marriage. Amer Math Monthly 69: pp9-15.

[3] Ishikawa S and Nakamura K (1979) The strategy-proof social choice functions. $J$ Math Econ 6: pp283-95.

[4] Maskin E (1985) The theory of implementation in Nash equilibrium: A survey. Hurwicz L, Schmeidler D and Sonnenschein H (eds) Social Goals and Social Organization. Cambridge University Press, Cambridge, pp 173-04

[5] Maskin E (1999) Nash equilibrium and welfare optimality. Rev Econ Stud 66: pp2338.

[6] Muller E and Satterthwaite M (1977) The equivalence of strong positive association and strategy-proofness. J Econ Theory 14: pp412-18.

[7] Shapley L and Scarf H (1974) On cores and indivisibilities. J Math Econ 1: pp23-27.

[8] Sönmez T (1999) Strategy-proofness and essentially single-valued cores. Econometrica 67: pp677-89.

\footnotetext{
${ }^{2}$ The complete definition of the problem includes initial endowments, which are superfluous for the present purpose thus dropped here.
} 
[9] Svensson L-G (1999) Strategy-proof allocation of indivisible goods. Soc Choice Welfare 16: pp557-67.

[10] Takamiya K (2001) Coalition strategy-proof and monotonicity in Shapley-Scarf housing markets. Math Soc Sciences 41: pp201-13.

[11] Takamiya K (2003) On strategy-proofness and essentially single-valued cores: A converse result. Soc Choice Welfare 20: pp77-83. 